\title{
Rastreio do Distress, o Sexto Sinal Vital em Oncologia: Dados Preliminares de Doentes Recém-Diagnosticados no Hospital CUF Porto
}

\section{Screening for Distress, the Sixth Vital Sign in Oncology: Preliminary Data of Newly Diagnosed Cancer Outpatients of Oporto Hospital CUF}

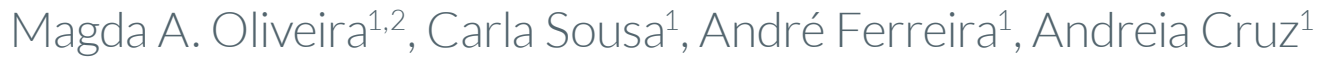

Autor Correspondente:

Magda A. Oliveira [magdaaoliveira@sapo.pt] Estrada da Circunvalação 341, 4100-180 Porto, Portugal

\section{RESUMO}

INTRODUÇÃO: A National Comprehensive Cancer Network (NCCN) define distress como uma experiência psicológica, social e espiritual desagradável que interfere na capacidade de lidar com a doença. Múltiplos estudos revelam que a prevalência oscila entre 30\% e 40\%. O seu não reconhecimento impossibilita a referenciação para cuidados psicossociais especializados, o que acarreta custos biopsicossociais e financeiros. Neste sentido, a NCCN propôs como medida, a implementação de rastreios de distress protocolados, com vista a avaliação sistemática dos doentes nos momentos-chave do processo terapêutico.

MATERIAL E MÉTODOS: Foram analisados os dados de 80 doentes oncológicos, avaliados em ambulatório no Hospital de Dia oncológico do Hospital CUF Porto, com recurso a uma ficha de dados clínicos e sociodemográficos e ao termómetro do distress e respetiva lista de problemas.

RESULTADOS: Os dados revelam um distress médio de 5,01, estando a maioria dos doentes no, ou acima do, ponto de corte 5. Os diagnósticos com distress mais elevado foram os tumores cabeça-pescoço, cerebrais e urogenitais. A fase da doença com níveis superiores foi a de recidiva, seguindo-se a de início de tratamento e a paliativa. Os problemas mais frequentes por categoria foram: preocupação com as tarefas domésticas, preocupação com os filhos, nervosismo/ansiedade, e problemas de sono. Dos doentes, 23,8\% foram referenciados para consultas de Psicologia/Psiquiatria após verbalizarem esta necessidade.

CONCLUSÃO: Os dados obtidos revelam níveis elevados de distress, adequadamente identificado e intervencionado quando desejado, em resultado da implementação deste programa de rastreio. Esta prática reforça os modelos preconizados que integram rastreio, tecnologia, referenciação e intervenção psicossocial apropriada às particularidades de cada doente.

PALAVRAS-CHAVE: Neoplasias/psicologia; Qualidade de Vida; Stress Psicológico 


\section{ABSTRACT}

INTRODUCTION: The National Comprehensive Cancer Network (NCCN) defines distress as an unpleasant psychological, social and spiritual experience that interferes with ability to cope with the disease. Multiple studies show that prevalence varies between $30 \%$ and $40 \%$. Its non-recognition makes it impossible to refer to specialized psychosocial care, which entails biopsychosocial costs and financial resources. In this sense, the NCCN proposed as a measure, the implementation of distress protocols, with a view to the systematic evaluation of patients at the therapeutic process.

MATERIAL AND METHODS: Data from 80 oncological patients were collected. Each of them was evaluated in the outpatient clinic at the Cancer Department on CUF Porto Hospital, using clinical and socio-demographic data and the Distress Thermometer and the respective list of problems.

RESULTS: Data show an average distress of 5.01, with most patients at or above the cutoff point 5 . The diagnoses with higher distress were head-neck, brain and urogenital tumors. The stage of disease with higher levels were relapsed, followed by treatment initiation and palliation. The most frequent problems by category were: preoccupation with domestic tasks, worry about children, nervousness/anxiety, and sleep problems. Of the patients, 23.8\% were referred to Psychology/Psychiatry consultations after verbalizing this need.

CONCLUSION: The data obtained reveal high levels of distress, adequately identified and intervened when desired, as a result of the implementation of this screening program. This practice reinforces the models already existents that cover screening, technology, referral, and psychosocial intervention appropriate to particularities of each patient.

KEYWORDS: Neoplasms/psychology; Quality of Life; Stress, Psychological

\section{INTRODUÇÃO}

Durante décadas, a medicina tradicional descurou a prestação de cuidados psicossociais aos doentes oncológicos e seus familiares, mesmo que muitas vezes fossem solicitados e apontados como imperativos. Todavia, esta realidade começou a modificar-se quando em 1997 a National Comprehensive Cancer Network (NCCN) reuniu um painel interdisciplinar de especialistas que se dedicou à análise desta questão, acabando por sugerir que a observação clínica não era suficiente como instrumento de avaliação, devendo recorrer-se, assim como na avaliação da dor, a uma questão única, sob a forma de escala analógica pontuada de 0 a 10, cuja finalidade era avaliar o nível de sofrimento emocional e as preocupações psicossociais relacionadas. ${ }^{1,2}$

O termo distress foi o conceito escolhido como aquele que melhor representaria a constelação de respostas psicossociais e preocupações associadas e, como aquele que mais se afastaria do estigma frequentemente implícito à sintomatologia psicológica/psiquiátrica. Após múltiplos estudos, a abordagem consensualmente validada traduziu-se até hoje na questão "Numa escala de 0 a 10, em que 0 é sem sofrimento e 10 é sofrimento extremo, qual a intensidade do seu sofrimento emocional na última semana, incluindo hoje?", sendo que, quando a resposta obtida se encontra acima do ponto de corte validado, a avaliação deve ser complementada. ${ }^{1,3,4}$

Com base nestes trabalhos, a Canadian Federal Government Public Health Agency apontou em 2004 o distress como o sexto sinal vital em Oncologia (depois do pulso, respiração, tensão arterial, temperatura e dor), sugerindo que passasse a ser avaliado por rotina pelos diferentes profissionais de oncologia que prestam cuidados ao doente oncológico e familiares, e intervencionado de acordo com as diretrizes práticas e clínicas para a gestão do distress que entretanto a NCCN havia definido e publicado sob a forma de handbook em 2006.1,5,6 A NCCN $(2013)^{7}$ acresceu ainda a ideia de que os programas de rastreios quando isolados não surtem os resultados esperados, devendo obedecer a um algoritmo de triagem que torne os resultados sustentáveis e perduráveis, o que requer o desenvolvimento de um plano assistencial por parte das unidades de Oncologia que permita alocar os doentes com scores elevados a consultas da especialidade. ${ }^{6}$

\section{O RASTREIO DO DISTRESS}

A NCCN (2002, p.6) $)^{8}$ definiu distress como "uma experiência emocional multifatorial desagradável de natureza psicológica (cognitiva, comportamental, emocional), social e/ou espiritual, que pode interferir com a capacidade de lidar efetivamente com o cancro, os seus sintomas físicos e o seu tratamento. $O$ distress estende-se ao longo de um contínuo que vai desde sentimentos comuns e normativos de vulnerabilidade, tristeza e medo, até problemas que podem tornar-se debilitantes tais como a depressão, ansiedade, pânico, isolamento social e crises existencial e espiritual". Quando na presença de níveis de distress que excedem o expectável ou que causam uma perturbação significativa no funcionamento do 
doente, este configura-se como diagnóstico psiquiátrico, destacando-se as perturbações de adaptação, as perturbações ansiosas e as perturbações depressivas.

Considerando a definição acima, entende-se que o distress experienciado na sequência de um diagnóstico de cancro e seus tratamentos, está intrinsecamente relacionado com um amplo leque de problemas de natureza prática, fisiológica, psicológica e espiritual e que, quando percecionado como elevado, está positivamente correlacionado com a perceção de uma qualidade de vida diminuída, com uma maior insatisfação com os cuidados de saúde prestados e com uma sobrevivência reduzida, sobretudo em situações de doença com estadiamento superior.4,10,11 Pelo facto da doença oncológica assumir várias trajetórias face ao prognóstico e tempo de tratamento e, face à multiplicidade de efeitos adversos possíveis, é recomendado que os pacientes sejam monitorizados tanto no diagnóstico e início de tratamentos, como por rotina ao longo de todo o processo terapêutico, de modo a garantir que recebem a assistência certa no momento adequado. ${ }^{12}$ Após a crise provocada pelo diagnóstico, frequentemente os níveis de distress diminuem, podendo ser novamente exacerbado perante os desafios que a doença e os tratamentos colocam. A esta flutuação dos níveis de distress estão associados períodos de vulnerabilidade acrescida, entre os quais: (a) o diagnóstico e as fases de transição, nomeadamente o início e fim dos tratamentos, a remissão, a sobrevivência, a recidiva, a doença avançada e o encaminhamento para cuidados paliativos; e, (b) os eventos relacionados com a doença, como as alterações na autoimagem, a dor, a perda da funcionalidade e as sequelas incapacitantes, o que sugere uma variância dos níveis de distress ao longo do curso da doença e tratamentos. 3,6,7,10

Procurando dar resposta às evidências da pertinência destes rastreios, a NCCN (2007)13 desenvolveu um instrumento de rastreio simples, rápido e robusto, designado de termómetro do distress (DT), para aplicação sistemática ao doente oncológico e/ou familiares, por parte dos diferentes elementos da equipa que intervêm junto dos mesmos. Este gold standard da avaliação consiste numa escala visual-analógica, pontuada de 0 a 10 , e acompanhada de uma lista de problemas de natureza múltipla (práticos, familiares, emocionais, espirituais, físicos), cuja finalidade é identificar e caraterizar rapidamente o sofrimento emocional e discriminar as necessidades de encaminhamento para os profissionais de saúde especializados, procurando-se atingir como fim último a melhor qualidade dos serviços de saúde prestados e consequentemente, a melhor qualidade de vida dos doentes e familiares. Por estas razões, este é um instrumento amplamente aceite e administrado, cumprindo os quesitos da NCCN e da APOS (American Psychosocial Oncology Society). ${ }^{2,7,14}$

De acordo com as guidelines da NCCN (2013), ${ }^{7}$ os doentes que merecem particular atenção por risco acrescido de distress elevado são aqueles em que há: antecedentes de perturbação psiquiátrica e/ou abuso de substâncias; antecedentes de depressão e/ou tentativa de suicídio; limitações cognitivas; comorbilidades severas; conflitos familiares/com cuidadores; suporte social percecionado como inadequado; filhos menores e/ou dependentes; problemas financeiros; barreiras na comunicação; um acesso limitado aos cuidados de saúde; história de abuso (físico, sexual); exposição a stressores concorrentes; maior inquietação espiritual e/ou religiosa; sintomatologia não controlada e, aqueles que são do sexo feminino ou vivem sozinhos.

\section{PAPEL DO RASTREIO}

Diferentes investigações demonstram que negligenciar a importância dos rastreios de distress tem um impacto negativo nos doentes e sistema de saúde: Ashbury e colaboradores (1998) mostraram que doentes com fadiga por distress não tratados recorriam cinco vezes mais ao serviço de saúde, iam duas vezes mais aos serviços de urgência e foram alvo de quimioterapias de terceira e quarta linha. ${ }^{15,16}$ Os estudos revelam ainda que há muito que o distress é identificado como um dos fatores mais significativos em Oncologia, por estar associado ao aumento do sofrimento emocional, a estilos de coping desadaptativos, a adesões terapêuticas mais pobres, a maior insatisfação com os cuidados prestados, a menor qualidade de vida, a mais dificuldades na comunicação e nos relacionamentos sociais, 3,10,17 a períodos mais longos de hospitalização, a pior controlo de sintomas, nomeadamente a dor, ${ }^{3}$ à manifestação de vontade de morrer e à pior qualidade de vida não só dos doentes mas também dos seus familiares e cuidadores. ${ }^{18}$ Pelo inverso, a identificação e referenciação adequadas, possibilitam que o doente seja alvo de intervenções psicossociais que reduzam de forma efetiva a sintomatologia, ${ }^{18}$ o que consequentemente reduz os custos do sistema de saúde. ${ }^{15,16}$ Estes dados corroboram o enorme fardo que é para o doente, para os familiares e para as instituições de saúde e seus profissionais, um sofrimento emocional não considerado e não tratado, contrapondo-se aos ganhos do rastrear, caraterizar e intervir precocemente no distress vivenciado. ${ }^{11}$

\section{DADOS DE PREVALÊNCIA}

Durante mais de 25 anos as investigações revelaram que pelo menos 25\% a 30\% dos doentes oncológicos apresenta uma perturbação emocional, de acordo com os manuais de diagnóstico psiquiátrico (DSM-IV, ICD-10) e com as ferramentas de rastreio do distress. ${ }^{19}$ No entan- 
to, existem dados em cujas taxas de prevalência de distress oscilam entre 35\% - 60\%, admitindo-se que possa verificar-se um subdiagnóstico associado à ausência de formação dos profissionais e programas de rastreios, o que tem implicações negativas na qualidade de vida, cronicidade do sofrimento emocional, satisfação com os cuidados prestados e tempo de sobrevivência. ${ }^{18,20}$ Uma das investigações mais importantes neste campo foi desenvolvida por Derogatis e colaboradores (1983) ${ }^{21}$ e incluiu 215 doentes oncológicos avaliados por entrevista e testes psicológicos que evidenciaram que 47\% dos doentes apresentavam um diagnóstico com base na DSM-III, sendo que 3\% apresentavam uma perturbação de personalidade e os restantes 44\% apresentavam uma perturbação clínica. Destes 44\%, 32\% tinham perturbações de adaptação, 6\% tinham perturbações afetivas major, 4\% tinham perturbações mentais orgânicas e 2\% tinham perturbações de ansiedade. Concluiu-se que mais de dois terços dos diagnósticos envolviam perturbações de adaptação reativas ao diagnóstico e tratamentos, tendo estes doentes uma boa resposta às intervenções psicológicas. Já em 2001, Zabora e colaboradores ${ }^{22}$ realizaram um estudo que integrou 4496 doentes com 14 diagnósticos diferentes, que revelou uma prevalência média de distress na ordem dos 35,1\%, oscilando entre $43,4 \%$ no cancro do pulmão e $29,6 \%$ no cancro ginecológico, sendo que os sete diagnósticos com scores mais elevados foram respetivamente: pulmão, sistema nervoso central, linfoma de Hodgkin, pâncreas, linfoma, fígado e cabeça-pescoço. Kendall e colegas (2011) ${ }^{3}$ reportaram por sua vez que na sua amostra de 1281 doentes oncológicos, 32\% dos mesmos reportava distress com uma intensidade entre 4 e 10 no DT, valor este que se situa no meio do intervalo 25\% - 50\% revelado em estudos precedentes. Grassi e colegas (2013), ${ }^{2}$ na revisão realizada observaram dados semelhantes, referindo que de acordo com muitos estudos desenvolvidos, a prevalência de distress oscila entre os 30\% e 40\%, podendo em alguns casos configurar-se como diagnóstico psiquiátrico, entre os quais se destacam as perturbações de adaptação, as perturbações ansiosas e as perturbações depressivas. Um estudo de Potash e Breitbart (2001) ${ }^{23}$ efetuado com uma amostra de doentes paliativos mostrou que a prevalência de níveis de distress significativos era superior a 58\%. Os dados empíricos demonstram ainda que entre os doentes que apresentam níveis mais elevados de distress, podemos distinguir dois grandes grupos: o grupo de doentes que cumprem critérios clínicos para doença psiquiátrica (25\%), e o grupo de doentes que não cumprem estes critérios mas que apresentam niveis de distress que interferem significativamente com o seu funcionamento habitual e com a sua qualidade de vida (15\% - 20\%). ${ }^{10}$

\section{BARREIRAS À REFERENCIAÇÃO}

Apesar de já muito documentada a importância e as mais-valias dos rastreios de distress, existem ainda muitas barreiras à referenciação dos doentes para serviços de apoio especializados, ${ }^{23}$ entre as quais se destaque a escassez de serviços e/ou recursos humanos especializados disponíveis, as limitações e constrangimentos financeiros, os longos períodos de espera pela primeira consulta, o tempo de consulta reduzido dos outros profissionais que não permite a identificação das necessidades psicossociais, a falta de sensibilização dos profissionais de oncologia para as questões psicossociais, a recusa dos pacientes em receber este suporte, ${ }^{2}$ e a falta de formação dos profissionais de saúde para detetar o distress. ${ }^{3}$ Mitchell, Vahabzadeh e Magruder (2011) ${ }^{24}$ sistematizaram as barreiras à identificação de distress sinalizadas pela NCCN (2013) ${ }^{7}$ com base em meta-análises publicadas anteriormente. Estas barreiras agrupam-se em três categorias: relacionadas com o doente - pertencer a uma minoria étnica, ser do género masculino, mostrar relutância em recorrer a apoio profissional, ocultar sintomas de determinada natureza e somatização das queixas psicológicas; relacionadas com os aspetos clínicos - pouca confiança e poucos conhecimentos dos clínicos, fragilidade da aliança terapêutica, tempo de consulta diminuto, consultas únicas com os clínicos, pouca sensibilidade para os aspetos psicossociais e avaliação pobre dos mesmos, relutância em diagnosticar ou sinalizar aspetos do foro psicológico/psiquiátrico; e, relacionados com a doença - apresentação atípica, contextos com baixa prevalência, severidade ligeira e presença de comorbilidade. Todavia, os dados apontam que as mulheres, as pessoas mais jovens, com um nível de escolaridade mais elevado, profissionalmente ativas, com um diagnóstico de cancro de mama e/ou com níveis clinicamente mais significativos de depressão, apresentam um recurso mais elevado aos serviços de apoio psicossocial. ${ }^{12,18}$ A sinalização e reflexão em torno destes obstáculos consolida a ideia de que as organizações de saúde devem reconhecer a complexidade da deteção e gestão das manifestações de sofrimento emocional, mobilizando-se para a supressão das barreiras à qualidade dos cuidados. Existem por isso, esforços internacionais para integrar estratégias e protocolos para rastreios por rotina em Oncologia. ${ }^{24}$

\section{MATERIAL E MÉTODOS}

Este estudo e os dados preliminares apresentados consistem na análise retrospetiva das informações recolhidas no âmbito do rastreio de distress implementado no Hospital de Dia Oncológico do Hospital CUF Porto. 


\section{PARTICIPANTES}

Foram analisados os dados de 80 doentes oncológicos, com mais de 18 anos e elegíveis para esta avaliação, que estavam a iniciar tratamento ambulatório em Hospital de Dia do Serviço de Oncologia do Hospital CUF Porto. Estes dados foram recolhidos durante 14 meses (2015-2016) junto de doentes oncológicos com diferentes diagnósticos e em diferentes estádios de doença, por uma psicóloga ou por um elemento da equipa de enfermagem do Hospital de Dia.

\section{INSTRUMENTOS DE AVALIAÇÃO}

Foram utilizados para a recolha de dados os seguintes meios: (a) uma ficha para colheita de dados sociodemográficos e clínicos com vista a uma melhor caraterização do doente; e, (b) o termómetro do distress e respetiva lista de problemas (Castro e Silva, 2007; tradução autorizada pela NCCN) para caraterização dos níveis de sofrimento emocional e reconhecimento de possíveis causas, mesmo que não diretamente relacionadas com o diagnóstico e tratamentos.

\section{PROCEDIMENTO E ANÁLISE ESTATÍ́STICA}

Foi feito um estudo retrospetivo da informação clínica recolhida no período mencionado acima. Os dados recolhidos foram introduzidos e tratados estatisticamente de acordo com programa estatístico SPSS (Stastistical Package for the Social Sciences).

\section{RESULTADOS}

\section{ASPETOS SOCIODEMOGRÁFICOS E CLÍNICOS DA AMOSTRA}

Foram analisados os dados dos instrumentos de avaliação de 80 pacientes, verificando-se que: a média de idades era de 59,6 anos (DP 11,6); 66,3\% ( $n=53$ ) dos participantes eram do sexo feminino e $33,8 \%(n=27)$ do sexo masculino; a maioria dos doentes era casada ( $n=$ $58,72,5 \%$ ), com dois filhos ( $n=30,37,5 \%$ ), com um trabalho qualificado ( $n=57,71,3 \%$ ), no momento não ativo ( $n=31,38,8 \%$ ); os diagnósticos mais prevalentes eram respetivamente por patologia: mama (24, 30\%), digestivos (22, 27,5\%) e pulmão (14, 17,5\%); para três doentes $(3,8 \%)$ esta era uma segunda neoplasia; 48 doentes (60\%) estavam numa fase de início de tratamentos, 11 $(13,8 \%)$ em fase de tratamentos, 13 (16,3\%) em recidiva e 8 (10\%) em fase paliativa; a maioria dos doentes estava proposto para uma combinação de estratégias terapêuticas ( $n=60,75,1 \%) ; 66$ doentes (82,5\%) nunca tinham tido acompanhamento por Psiquiatria e 72 (90\%) nunca tiveram consultas de Psicologia.

\section{DADOS DO RASTREIO}

A análise dos dados contidos no termómetro do distress revelaram que: a média de distress na amostra era de 5,01 (DP 2,85); 34 doentes (42,5\%) encontravam-se abaixo do ponto de corte para a população portuguesa (score 5), 35 (43,8\%) responderam entre 5 e 8 e, 11 $(13,8)$ tinham níveis severos de distress (9 ou 10); as médias por diagnóstico foram: cabeça-pescoço 7,50, tumores cerebrais 6,00, urogenitais 5,78, mama 5,67, pulmão 4,64, digestivos 4,18, onco-hematologia 4,14; as médias de distress por fase da doença foram: recidiva 5,3, início de tratamentos 5,00, em fase paliativa 4,88 e, em tratamento 4,82; não foram identificadas correlações estatisticamente significativas entre os níveis de distress e o tempo decorrido pós-diagnóstico (r.15; p .17) e entre os mesmos e a idade dos participantes ( $r$.18; $p$.11); relativamente à lista de problemas, os mais e menos representados foram (Tabela 1):

TABELA 1. Lista de Problemas.

\begin{tabular}{|c|c|c|}
\hline Lista de Problemas & Mais Frequentes & Menos Frequentes \\
\hline Problemas Práticos & $\begin{array}{l}\text { Tarefas domésticas } 22(27,5 \%) \\
\text { Preoc. económicas } 21(26,3) \\
\text { Cuidado dos filhos } 20(25,0 \%)\end{array}$ & Transportes e deslocações 12 (15\%) \\
\hline Problemas Familiares & Filhos $29(36,3 \%)$ & Marido $22(27,5 \%)$ \\
\hline Problemas Emocionais & $\begin{array}{l}\text { Nerv./Ansiedade } 66 \text { (82,5\%) } \\
\text { Preocupações } 62 \text { (77,5\%) } \\
\text { Tristeza } 54(67,5 \%)\end{array}$ & Perda de interesse 28 (35\%) \\
\hline Problemas Espirituais & Sim $10(12,5 \%)$ & Não $70(87,5)$ \\
\hline Problemas Físicos & $\begin{array}{l}\text { Sono } 50 \text { (62,5\%) } \\
\text { Fadiga e cansaço } 41(51,3 \%) \\
\text { Aspeto físico } 39(48,8 \%) \\
\text { Dores } 38(47,5 \%)\end{array}$ & Febre $(3,8 \%)$ \\
\hline
\end{tabular}


Quando questionados no final da avaliação, 19 doentes (23,8\%) mostrou vontade em ter acompanhamento psicológico/psiquiátrico especializado e 61 (76,3\%), mesmo que com scores elevados não aceitou acompanhamento especializado.

\section{DISCUSSÃO}

É consensual que apesar de todos os recursos já existentes, ainda hoje o cancro é visto como uma doença cujo significado é ameaçador para a maioria das pessoas, não só pelo risco de morte, mas também pelo facto de romper com a estabilidade prévia da vida e rotina do doente, dos seus projetos de vida e das relações e papéis desempenhados. Por esta razão, compreende-se que estejam expostos a elevados níveis de sofrimento emocional (distress), com causas múltiplas e flutuantes ao longo da trajetória da doença, e com implicações várias.

Os dados recolhidos nesta amostra revelam níveis de distress superiores aos encontrados na maioria dos estudos apresentados na revisão acima, sendo que 57,6\% (n =46) dos doentes estudados tinha scores iguais ou superiores a 5, que corresponde ao ponto de corte do distress para a população portuguesa.

Outro dado interessante prende-se com o facto de apesar dos dados mostrarem que pouco mais de 10\% dos doentes são referenciados para cuidados psicossociais, ${ }^{16}$ na presente amostra, verificou-se que 23,8\% dos doentes ( $n=19$ ) manifestou vontade de suporte psicossocial, tendo sido encaminhado neste sentido. A percentagem mais elevada de doentes propostos para intervenção psicossocial revelou-se superior à encontrada em alguns estudos, o que pode ser um indicador dos benefícios do rastreio e da disponibilidade de recursos que a instituição oferece.

Os níveis elevados de distress encontrados nesta amostra podem também estar relacionados com dados que demonstram a relação entre níveis de distress aumentados e a presença de uma maior perceção de incapacidade, doença avançada e/ou perceção de dor. ${ }^{16}$ A literatura aponta ainda que a fadiga não tratada, a dor não controlada e a presença de níveis significativos de ansiedade, têm um significativo contributo para o empobrecimento da qualidade de vida, ${ }^{12}$ o que corrobora o encontrado nesta população.

Embora se encontrem algumas similaridades, esta amostra difere de estudos anteriores relativamente aos diagnósticos com maior prevalência. Carlson e Bultz (2004), ${ }^{16}$ revelaram que as patologias com maiores níveis de distress eram respetivamente: pulmão, tumores cerebrais, linfoma de Hodgkin, pâncreas, linfoma, cabe- ça-pescoço, mama, leucemia, melanoma, cólon, próstata e finalmente ginecológico. Na presente amostra parece haver alguma inversão destes dados.

Relativamente aos problemas identificados, o predomínio de aspetos psicossociais vai de encontro ao esperado, assim como a evidência da fadiga/cansaço, a dor e as mudanças no aspeto físico também estão sublinhadas como algumas das causas mais desencadeadoras de sofrimento no doente oncológico.

\section{CONCLUSÃO}

A realidade do cancro e seus tratamentos pode ser extremamente stressora para o doente, podendo despoletar medo, incerteza, sofrimento emocional e necessidades psicossociais, precipitantes de elevado distress, que quando não tratado pode conduzir a várias implicações. ${ }^{18}$ Todavia, apesar das recomendações e evidência empírica a suportar a importância da adoção de políticas de rastreio do distress sistemáticas e as limitações implícitas a uma observação clínica desapoiada, são poucos os programas e entidades que avaliam por rotina o distress dos doentes oncológicos e, são menos ainda aqueles que integraram uma robusta componente de investigação e/ou avaliação complementar. ${ }^{10,25}$

Os dados científicos atuais revelam a necessidade da realização de mais ensaios clínicos que aprofundem as investigações acerca dos efeitos do rastreio e tratamento atempado e adequado do distress sinalizado. Com a designação de sexto sinal vital, abriu-se a oportunidade para uma abordagem mais compreensiva da prestação de cuidados oncológicos, trazendo novos e interessantes desafios para a Oncologia e para a Psico-Oncologia em particular, na busca de respostas que permitam usar a ciência e os recursos existentes para incrementar a qualidade dos cuidados prestados. ${ }^{25}$

A estratégia específica que cada centro oncológico determina para garantir o tratamento adequado dos doentes em sofrimento emocional deverá variar de acordo com as particularidades dos doentes de cada centro e de acordo os recursos humanos e técnicos dos mesmos. Contudo, a NCCN (2013) ${ }^{7}$ recomenda a prestação integrada e personalizada de cuidados que contemple a dimensão psicossocial, o que apesar de ser um potenciador de resultados de saúde positivos para o doente e instituições de saúde, não deixa de ser concomitantemente um desafio para as segundas. ${ }^{6}$

No que concerne ao tratamento, a intervenção psicoterapêutica é considerado o tratamento de eleição, sobretudo perante situações de perturbações de adaptação, tendo como principais objetivos reduzir a severidade 
dos stressores e sintomas, reforçar e potenciar as estratégias de coping adaptativas e fortalecer a rede de suporte social e sua utilização pelo doente e familiares. ${ }^{10}$

CONFLITOS DE INTERESSE: Os autores declaram não ter qualquer conflito de interesse na realização do presente trabalho.

FONTES DE FINANCIAMENTO: Não houve qualquer fonte de financiamento na realização do presente trabalho.

CONFIDENCIALIDADE DOS DADOS: Os autores declaram ter seguido os protocolos da sua instituição acerca da publicação dos dados de doentes.

PROTEÇÃO DE PESSOAS E ANIMAIS: Os autores declaram que os procedimentos seguidos na elaboração do presente trabalho estão em conformidade com as normas das comissões de investigação clínica e de ética, bem como da declaração de Helsínquia e da Associação Médica Mundial.

CONFLICTS OF INTEREST: The authors declare that they have no conflicts of interest.

FINANCIAL SUPPORT: This work has not received any contribution, grant or scholarship.

CONFIDENTIALITY OF DATA: The authors declare that they have followed the protocols of their work center on the publication of data from patients.

PROTECTION OF HUMAN AND ANIMAL SUBJECTS: The authors declare that the procedures followed were in accordance with the regulations of the relevant clinical research ethics committee and with those of the Code of Ethics of the World Medical Association (Declaration of Helsinki).

\section{REFERÊNCIAS}

1. Holland JC, Bultz BD, National Comprehensive Cancer Network (NCCN). The NCCN Guideline for Distress Management: a Case for Making The Distress the Sith Vital Sign. J Natl Compr Canc Netw. 2007;5:3-7.

2. Grassi L, Johansen C, Annunziata MA Capovilla E, Costantini A, Gritti P, et al. Screening for distress in cancer patients - a multicenter nationwide study in Italy. Cancer. 2013;119:171421.

3. Kendall J, Glaze K, Oakland J, Hansen J, Parry C. What do 1281 distress screeners tell us about cancer patients in a community cancer center? Psychooncology. 2011;20:594600.

4. Mitchell AJ. Screening for cancer-related distress: when is implementation successful and when is it unsuccessful? Acta Oncol. 2013;52:216-24.

5. Rebalance Focus Action Group. A positive paper: screening key indicators in cancer patients: pain as $5^{\text {th }}$ vital sign and emotional distress as $6^{\text {th }}$ vital sign. Can Strategy Cancer Control Bull. 2005;7:4.

6. Pirl WF, Fann JR, Greer JA, Braun I, Deshields T, Fulcher C, et al. Recommendations for the implementation of distress screening programs in cancer centers: report from the American Psychosocial Oncology Society (APOS), Association of Oncology Social Work (AOSW), and Oncology Nursing Society (ONS) joint task force. Cancer. 2014;120:2946-54.

7. National Comprehensive Cancer Network. NCCN Clinical Practice Guidelines in Oncology: Distress Management. Version 3.2013. Fort Washington:NCCN; 2013.

8. National Comprehensive Cancer Network: Practice Guidelines in Oncology, Version 1.2002: Distress Management. Fort Washington: NCCN; 2002.

9. Oliveira M, Castro S, Almeida S, Moutinho S. Cancro, Vidas em Reconstrução - Uma Abordagem Psicológica da Doença, suas Problemáticas e Emoções. Porto: LivPsic;2012.

10. Carlson LE, Waller A, Groff SL, Zhong L, Bultz BD. Online screening for distress, the $6^{\text {th }}$ vital sign, in newly diagnosed oncology outpatients: randomised controlled trial of computerized vs personalized triage. Br J Cancer. 2012;107:617-25.

11. Carlson LE, Waller A, Mitchell AJ. Screening for distress and unmet needs in patients with cancer: review and recommendations. J Clin Oncol. 2012;30:1160-77.

12. Waller A, Williams A, Groff SL, Bultz B, Carlson LE. Screening for Distress, the Sixth Vital Sign: Examining Self-Referral in People with Cancer Over a One-year Period. Psychooncology. 2013;22:388-95.

13. National Comprehensive Cancer Network. Clinical Practice Guidelines in Oncology: Palliative Care. Fort Washington: NCCN; 2007.

14. Donovan KA, Grassi L, McGinty HL, Jacobsen PB. Validation of the Distress Thermometer Worldwide: State of Science. Psychooncology. 2014;23:241-50.

15. Simpson JS, Carlson LE, Beck CA, Patten S. Effects of a Brief Intervention on Social Support and Psychiatric Morbidity in Breast Cancer Patients. Psychooncology. 2002;11:282-94.

16. Carlson LE, Bultz BD. Efficacy and Medical Cost Offset of Psychosocial Interventions in Cancer Care: Making the Case for Economic Analyses. Psychooncology. 2004;13:837-49.

17. Gil F, Grassi L, Travado L, Tomamichel M, Gonzales J. Use of distress and depression thermometers to measure psychosocial morbidity among southern European cancer patients. Supp Care Cancer. 2005;13:600-6.

18. Merckaert I, Libert Y, Messin S, Milani M, Slachmuylder JL, Razavi D. Cancer patients desire for psychological support: prevalence and implications for screening patients' psychological needs. Psychooncology. 2010;19:141-9.

19. Grassi L, Rossi E, Caruso R, Nanni M, Pedrazzi S, Sofritti S, et al. Educational intervention in cancer outpatients clinics on routine screening for emotional distress: an observational study. Psychooncology. 2011;20:669-74.

20. Giese-Davis J, Waller A, Carlson LE. Screening for distress, the $6^{\text {th }}$ vital sign: common problems in cancer outpatients over one year in usual care: associations with marital status, sex, and age. BMC Cancer. 2012;2:441-53.

21. Derogatis LR, Morrow GR, Fetting D, Penman D, Piasetsky $\mathrm{S}$, Schmale AM, et al. The prevalence of psychiatric disorders among cancer patients. JAMA. 1983;249: 51.

22. Zabora J, Brintzenhofeszoc K, Curbow B, Hooker C, Piantodosi S. The Prevalence of Psychological Distress by Cancer Site. Psychooncology. 2001;10:19-28.

23. Potash M, Breitbart W. Affective disorders in advanced cancer. Hematol Oncol Clin North Am. 2002;16:671-700.

24. Mitchell AJ, Vahabzadeh A, Magruder K. Screening for distress and depression in cancer settings: 10 lessons from 40 years of primary care research. Psychooncology. 2011;20:572-84.

25. Bultz B, Johansen C. Screening for distress, the 6th vital sign: where are we, and where are we going? Psychooncology. 2011;20:569-71. 